protocol so that the collective experience of many institutions will contribute to the further understanding and better management of such cases. Much more needs to be known if women with renal transplants are to be carefully selected to enjoy pregnancy and parenthood with maximum benefit to themselves and their offspring. We doubt, however, if a decision to undertake a transplant should ever be based on a desire for parenthood.

J M Davison

T LIND

MRC Reproduction and Growth Unit, Princess Mary Maternity Hospital

D N S KERR

Department of Medicine,

Royal Victoria Infirmary,

' Davison, J M, Lind, T, and Uldall, P R, British fournal of Obstetrics and Gynaecology. In press.

Merkatz, J R, et al, Ұournal of the American Medical Association, 1971, 216, 1749.

\section{Hypoglycaemia in children undergoing} adenotonsillectomy

SIR,-Those constantly concerned with eliminating the risks associated with operations on the tonsils and adenoids will be grateful to $\mathrm{Dr}$ C J H Kelnar for his contribution (27 March, p 751). He states, however, "It is not unreasonable to suppose that some of the 15 to 20 children who die each year after adenotonsillectomy die from the effects of unrecognised hypoglycaemia." It may well be that some children die from this cause, but the total number of deaths per annum is very much less than quoted. In the five years 1964-8 the annual number of deaths from this operation had dropped to little less than seven and in 1970 figures from the Office of Population Censuses and Surveys showed that there was but one death and that from adenoidectomy without tonsillectomy.

In recent years improved management and a steady reduction in the number of operations have combined to reduce the mortality rate to very small proportions. Nevertheless, the utmost vigilance is still required, which should certainly take into account the important factor publicised by $\mathrm{Dr}$ Kelnar.

London W1

STUART MaWson

\section{Thyrotoxicosis induced by iodine in food}

SIR,-I enjoyed reading the authoritative paper by Drs J C Stewart and G I Vidor (14 February, p 372) in which they pointed out that increased frequency of thyrotoxicosis was observed after addition of iodine to the diet in northern Tasmania. It has been suggested $^{1}$ that the development of endemic goitre is related to a balance between thyroidstimulating hormone (TSH) production and thyroid size. Iodine deficiency thus leads to decreased thyroid hormone production, which in turn causes increased TSH secretion and increased thyroid size until there is enough thyroid tissue to trap a proportion of circulating iodine large enough to maintain normal hormone levels. Any increase in iodine intake in such persons thus causes a trend towards thyrotoxicosis because the thyroid is adjusted to a higher than normal degree of iodine utilisation.

In a goitre region with iodine deficiency any increase in iodine intake must thus lead to an initial increase in the frequency of thyrotoxicosis, as was shown by the authors. If, however, iodine intake in the area becomes permanently adequate through iodine prophylaxis the frequency of thyrotoxicosis would eventually decrease. The increased incidence of thyrotoxicosis after iodine is added to food is thus a consequence of iodine deficiency.

An accumulation of thyrotoxicosis patients in the generation in which iodine prophylaxis is begun seems to be the price to pay for normal thyroid physiology in the following generations. When basal iodine intake is sufficient to maintain normal hormonal balance occasiona increases in iodine intake should not cause any increased frequency of thyrotoxicosis. In other words, bread and milk would still be preferable to cakes and ale-albeit not for the goitrous. Peter WAHLBERG Department of Internal Medicine, Aland Central Hospital,

Mariehamn,

Becker, F O, in Year Book of Endocrinology 1974, p 198.
Chicago, Year Book Medical Publishers, 1974.

\section{IUD and congenital malformation}

SIR,-One must never dismiss reports of possible iatrogenic damage to the developing fetus. But the two cases described by $\mathrm{Dr}$ Herbert Barrie (28 February, p 488) provide little support for a cause-and-effect link between copper-containing intrauterine devices (IUDs) and limb-reduction defects. As suggested by Dr Robert Snowden (27 March, p 770), conception and organogenesis in the second case may have occurred after expulsion of the IUD. Moreover, the lead content $\left(0 \cdot 01-1{ }_{0}\right)$ of the Gräfenberg ring in the first case may have caused the malformation rather than the copper-if the association was not coincidental. The chief problem is that the relevant denominator is unknown-that is, the size of the population fitted with Gräfenberg rings and the number of in-situ pregnancies occurring. In Dr Snowden's study ${ }^{1}$ these figures are available, and it is reassuring that no congenital abnormalities were reported among the $21^{\circ}$ of 317 pregnancies reaching viability.

What about devices which contain much more (about $90 \mathrm{mg}$ ) of copper? The following results are by courtesy of the medical department of Searle Laboratories, High Wycombe, and relate solely to the Copper-7 device (Gravigard). In two international studies there were 20684 insertions, and 714 pregnancies with Copper-7 devices in situ at conception have been reported. Of these, 167 reached viability, $8 \%$ were lost to follow-up, and the remainder were ectopic or ended in spontaneous or induced abortion. Normal babies resulted from 159 pregnancies. No details were given about three infants and the remaining five had the following malformations: (1) permanent bald spot; (2) bilateral congenital eyelid ptosis; (3) fatty tumour on the back; (4) congenital dislocation of hip (breech delivery); (5) lumbosacral meningomyelocele and bilateral talipes-neonatal death at seven days. If the three doubtful cases are included the incidence of congenital defects was eight out of 167 or $4.8 \%$ (very close to the figure quoted by Dr Barrie as the expected rate).

Note that no limb-reduction defects were reported. However, in addition to the above cases from a defined population there have been 23 pregnancies reaching viability and reported spontaneously by doctors to Searle's -all from the United Kingdom. Nineteen normal infants were reported and of the four cases of congenital abnormality one was that described by Miss P C Leighton and her colleagues (24 April, p 949) with multiple limb-reduction deformities. This is the only such case reported in Britain since the Gravigard was first marketed in September 1972. The size of the population at risk from which the case comes is unknown. (A very approximate indication may be obtained, based on the fact that about 376000 of these devices have been sold within the British Isles.)

In conclusion, I would agree with the last paragraph of Dr Barrie's article. The likelihood of spontaneous abortion, which may be septic, ${ }^{2}$ and of other dangerous complications of pregnancy and delivery ${ }^{4}$ is such that most authorities would agree that "serious consideration should be given to terminating pregnancies with a retained intrauterine device" on maternal grounds. But on the figures given here the teratogenic risk to the fetus if a Copper-7 device was in use would not be added grounds for legal abortion.

JohN Guillebaud

Nuffield Department of Obstetrics

and Gynaecology,

Oxford

Snowden, R, FPA Medical Newsletter, No 59, 1976, Eisinger, S H, American fournal of Obstetrics and Gynecology, 1976, 124, 393.

Vessey, M P, et al, Lancet, 1974, 1, 495.

teven, J D, and Fraser, I S, Fournal of Obstetrics and 282 .

Control of menstrual bleeding during haemodialysis

SIR,-Over the past two years a new intrauterine device (IUD) releasing progesterone (Progestasert) has been used for contraception. ${ }^{1-3}$ During these studies it was observed that total menstrual blood loss decreased and removals of the device for bleeding were reduced.

Patients with renal disease awaiting transplantation and undergoing haemodialysis often experience heavy and troublesome menstrual periods. Control of these periods with continuous hormone therapy (usually a combined oestrogen and progestogen pill) often gives rise to side effects. In an attempt to reduce these side effects we have used the progesterone IUD ror menstrual cycle control in four patients awaiting renal transplantation. All four had chronic glomerulonephritis with varying periods of amenorrhoea during the progression of their kidney disease. With dialysis all developed heavy periods requiring treatment. Norethisterone $30 \mathrm{mg} /$ day controlled two patients' bleeding but gave other unpleasant side effects, such as a bloated feeling and water retention. On reduction of the dose to $10 \mathrm{mg} /$ day both experienced troublesome breakthrough bleeding. Of the other two patients, one had heavy periods with norethisterone and the other severe breakthrough bleeding. All four patients experienced troublesome menstrual side effects when combined oral contraceptive preparations were used.

The progesterone IUD was easily inserted in all four patients. Following insertion all four had regular menstrual cycles with accept- 